\title{
Human umbilical cord blood-derived mesenchymal stem cell transplantation attenuates severe brain injury by permanent middle cerebral artery occlusion in newborn rats
}

\author{
Eun Sun Kim', So Yoon Ahn', Geun Ho Im², Dong Kyung Sung ${ }^{3}$, Ye Rim Park ${ }^{3}$, Seo Hui Choi ${ }^{4}$, Soo Jin Choi ${ }^{5}$, Yun Sil Chang ${ }^{1,3}$, \\ Wonil Oh', Jung Hee Lee ${ }^{2}$ and Won Soon Park ${ }^{1,3}$
}

\begin{abstract}
BACKGROUND: Severe brain injury induced by neonatal stroke causes significant mortality and disability, and effective therapies are currently lacking. We hypothesized that human umbilical cord blood (UCB)-derived mesenchymal stem cells (MSCs) can attenuate severe brain injury induced by permanent middle cerebral artery occlusion (MCAO) in rat pups.
\end{abstract}

METHODS: After confirming severe brain injury involving more than $50 \%$ of the ipsilateral hemisphere volume at $1 \mathrm{~h}$ after MCAO using diffusion-weighted magnetic resonance imaging (MRI) in postnatal day (P)10 rats, human UCB-derived MSCs were transplanted intraventricularly. The brain MRI was evaluated periodically up to $28 \mathrm{~d}$ after MCAO (P38). Sensorimotor function and histology in the peri-infarct tissues were evaluated at the end of the experiment.

RESULTS: Severe brain injury induced by permanent MCAO resulted in decreased survival and body weight gain, increased brain infarct volume as measured by MRI, impaired functional tests such as the rotarod and cylinder test, and histologic abnormalities such as increased terminal deoxynucleotidyl transferase nick-end labeling, reactive microglial marker, and glial fibrillary acidic protein-positive cells in the penumbra. All of these abnormalities were significantly improved by MSC transplantation $6 \mathrm{~h}$ after MCAO.

CONCLUSION: These results suggest that human UCB-derived MSCs are a promising therapeutic candidate for the treatment of severe perinatal brain injury including neonatal stroke.

N eonatal stroke is a serious perinatal brain injury causing significant mortality and long-term neurological morbidity in survivors. Effective treatments are currently lacking (1). The incidence of neonatal stroke has increased to 1 of 2,300 to 4,000 full-term neonatal births due to recent improvements in neurodiagnostic imaging techniques $(1,2)$. Because neonatal stroke frequently occurs in the middle cerebral artery (MCA) region and is mostly of ischemic origin (1), MCA occlusion (MCAO) models in neonatal rodents have been used for pathophysiological studies of focal ischemic stroke in immature brains $(3,4)$.
Therapeutic interventions such as hypothermia that are known to be effective for perinatal asphyxial brain injury could be applied to neonatal stroke because of similarities in their pathophysiology $(5,6)$. However, even therapeutic hypothermia was not effective against a severe type of neonatal brain injury $(6,7)$. Therefore, the development of new therapeutic modalities to improve the prognosis of this severe type of neonatal brain injury resulting from either perinatal asphyxia or neonatal stroke is an urgent issue.

Recently, the neuroprotective effects of exogenously administered mesenchymal stem cells (MSCs) have been reported in adult stroke models of MCAO $(8,9)$. Umbilical cord blood (UCB) is considered the best source of MSCs as compared with other sources such as bone marrow or adipose tissue because it is easy to obtain. We have shown that the xenotransplantation of human UCB-derived MSCs protects against disorders such as bronchopulmonary dysplasia $(10,11)$ and acute respiratory distress syndrome (12). Furthermore, there are ongoing clinical trials of MSC transplantation, including phase I clinical trials for bronchopulmonary dysplasia (13) and cerebral palsy (14). Overall, these findings suggest that MSC transplantation may be a novel therapeutic modality for attenuating severe brain damage after neonatal stroke. However, the results obtained in adults cannot be directly extrapolated to neonatal medicine due to the dramatic differences in the maturational stage and pathophysiology of perinatal and adult brains. Therefore, favorable experimental results that show that MSC transplantation can protect against severe MCAO in a newborn animal model will be necessary for future translation in the treatment of neonatal stroke patients.

In this study, we determined the therapeutic efficacy of human UCB-derived MSC transplantation in attenuating the severe brain injury induced by permanent $\mathrm{MCAO}$ in newborn rats. Human UCB-derived MSCs were transplanted only after confirming the existence of severe brain injury induced by permanent MCAO using diffusion-weighted imaging (DWI) of brain magnetic resonance imaging (MRI). The therapeutic

\footnotetext{
The first two authors share first authorship.

'Department of Pediatrics, Samsung Medical Center, Seoul, South Korea; ${ }^{2}$ Department of Radiology, Samsung Medical Center, Seoul, South Korea; ${ }^{3}$ Samsung Biomedical Research Institute, Sungkyunkwan University School of Medicine, Seoul, South Korea; ${ }^{4}$ Department of Pediatrics, Samsung Changwon Medical Center, Sungkyunkwan University School of Medicine, Changwon, South Korea; ${ }^{5}$ Biomedical Research Institute, Medipost, Seoul, South Korea. Correspondence: Won Soon Park (wonspark@skku.edu) 
efficacy of the MSC transplantation was evaluated using serial brain MRI monitoring in vivo, sensorimotor function tests such as the rotarod and cylinder tests, and histology in the peri-infarct area by glial fibrillary acidic protein (GFAP), reactive microglial marker (ED-1), neuronal-specific nuclear protein (NeuN), and terminal deoxynucleotidyl transferase nickend labeling (TUNEL) staining.

\section{RESULTS}

\section{Survival Rates and Body Weights}

Severe brain injury induced by permanent MCAO (MCAO + phosphate-buffered saline (PBS)-treated group (MC)) reduced the survival rate to $40 \%$ at $28 \mathrm{~d}$ post-MCAO (postnatal day (P)38) as compared with the $100 \%$ survival rate of sham + PBS-treated group (NC). This reduced survival rate observed in MC improved to $83 \%$ after MSC transplantation (MCAO + human UCB-derived MSC-treated group (MM)) (Figure 1a).

Although the body weight at P10 was not significantly different among the three experimental groups, the body weight at $28 \mathrm{~d}$ post-MCAO (P38) in MC was significantly lower compared with that observed in NC. This decrease in body weight gain observed in MC was significantly improved with MSC transplantation (MM) (Figure 1b).

\section{Serial Brain MRI and Injury Assessment}

Serial in vivo brain MRI demonstrated the extension of the severe brain injury beyond the ipsilateral cortex into the striatum, thalamus, or hippocampus, as evidenced by the hyperintense areas in the diffusion-weighted MRI performed $1 \mathrm{~h}, 6 \mathrm{~h}$, and $3 \mathrm{~d}$ post-MCAO (on P10 and P13) and by T2-weighted MRI performed 3, 7, and $28 \mathrm{~d}$ post-MCAO (on P13, P17, and P38, respectively) (Figure $2 \mathrm{a}-\mathbf{d}$ ). The hyperintense areas in the DWI were closely correlated with the hyperintense areas in the T2-weighted imaging at day 3 after injury $\left(r^{2}=0.93, P<0.01\right)$ (data not shown).

Initially, at 1-h post-MCAO, the ipsilateral to contralateral hemispheric intact brain volume ratio was low in $\mathrm{MC}$, and remained significantly low as compared with that observed in NC throughout the experiment (Figure 2e). Although

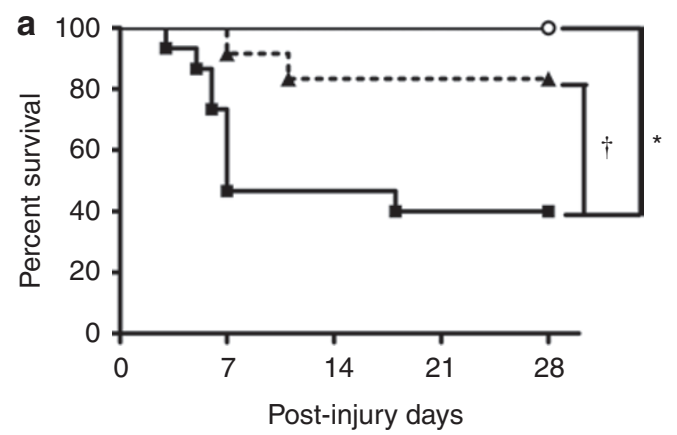

the initial brain volume ratio at 1 -h post-MCAO was similar between $\mathrm{MC}$ and MM, the intact volume ratio in MM was significantly higher as compared with $\mathrm{MC}$ at 3, 7, and 28 days post-MCAO (P13, P17, and P28, respectively), which suggests that MSC transplantation significantly attenuated the severe brain injury induced by MCAO.

$\mathrm{T} 2^{*}$-weighted imaging revealed low-signal-intensity areas, reflecting the presence of transplanted super-paramagnetic iron oxide-tagged MSCs, and the persistence of this signal in the peri-infarct area until day 28 days post-MCAO (P38) is suggestive of MSC migration to the injured area in MM (Figure 2a-d), but not in NC or MC.

\section{Functional Tests}

Both the latency to fall in the rotarod test and the limb-use asymmetry in the cylinder test measured on the 28th day postMCAO (P38) were significantly lower in MC as compared with $\mathrm{NC}$; these decreased functional test results were improved with MSC transplantation (MM) (Figure 3). However, the improvement in the cylinder test results was not statistically significant. In the rotarod test, latency to fall was significantly lower in MC than in NC. MSC transplantation (MM) attenuated this decrease in latency time before falling on the 28th day postMCAO (P38) (Figure 3a). In the cylinder test, limb-use asymmetry was significant in MC; however, MSC transplantation was not different from NC at $28 \mathrm{~d}$ post-MCAO (P38) (MM) (Figure 3b).

\section{Histological Evaluation and Donor Cell Presence}

The hematoxylin and eosin-stained brain tissue of the rats at $28 \mathrm{~d}$ post-MCAO (P38) demonstrated significant tissue loss in the affected hemisphere as compared with that of $\mathrm{NC}$ (Figure 4a). This effect was less obvious in MM as compared with MC, which correlates with the day 28 brain MRI results obtained shortly before the animals were killed. Microscopic examination revealed that the cortical areas of the bregma and the hippocampus were intact in NC, whereas significant cortical loss was observed in MC (Figure 4b). These morphological changes and the associated neuronal loss were attenuated in MM as compared with MC (Figure 4a,b).

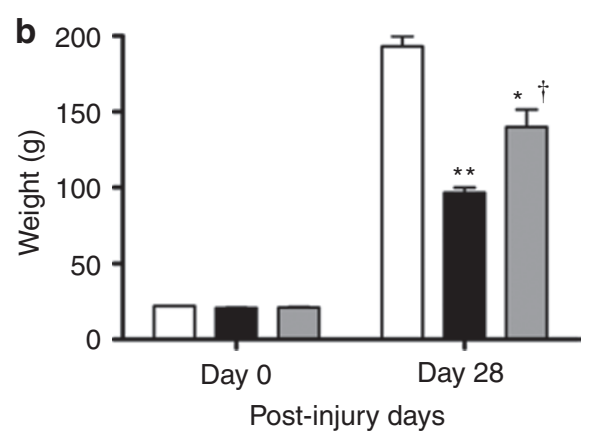

Figure 1. Survival rates and weight changes. (a) Survival rates in each group were determined using Kaplan-Meier analysis followed by a log-rank test. (b) Weight changes from the day of middle cerebral artery occlusion (MCAO) until the end of the study. Circles and white bars represent the NC, squares and black bars represent the $\mathrm{MC}$, and triangles and gray bars represent the MM group. MC, MCAO + PBS-treated group; MM, MCAO + human umbilical cord blood (UCB)-derived mesenchymal stem cell (MSC)-treated group; NC, sham + phosphate-buffered saline (PBS)-treated group (NC, $n=5 ;$ MC, $n=$ $15 ; \mathrm{MM}, n=12) .{ }^{*} P<0.05$ vs. NC group, ${ }^{* *} P<0.01$ vs. NC group, and ${ }^{+} P<0.05$ vs. MC group. 
a

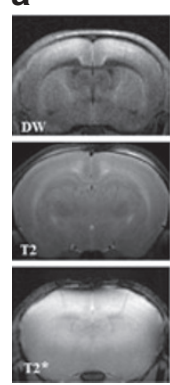

c

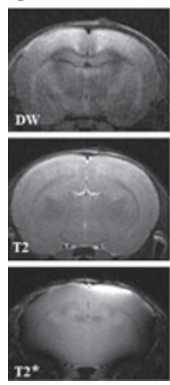

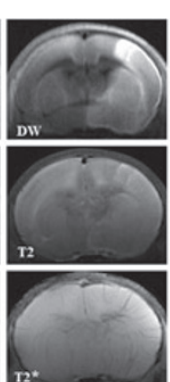
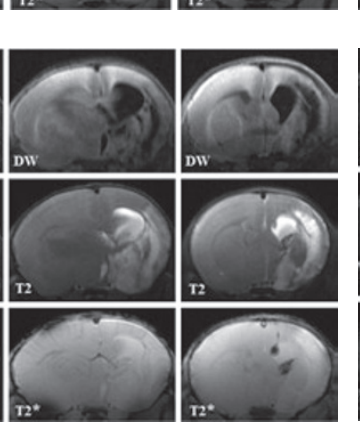
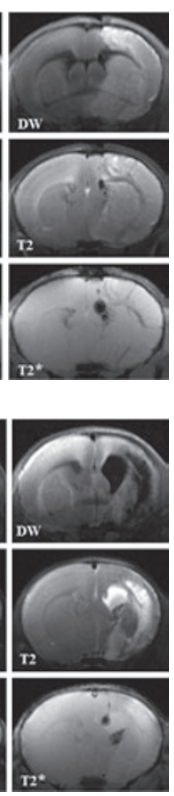

b

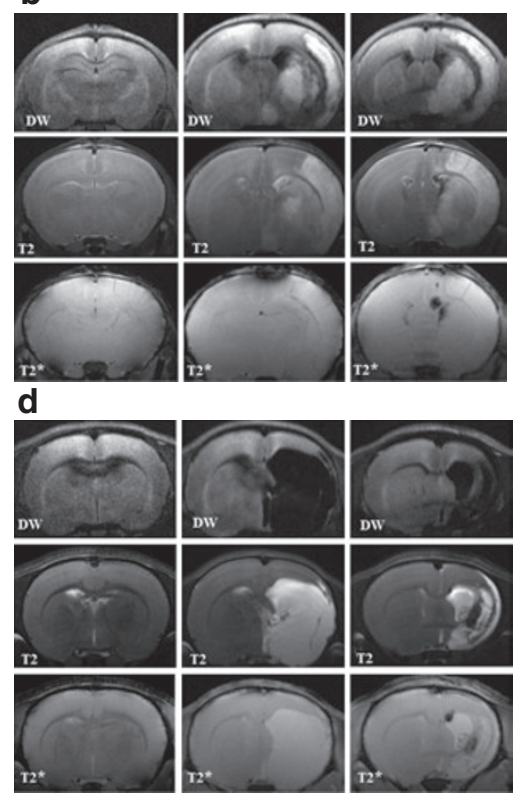

e

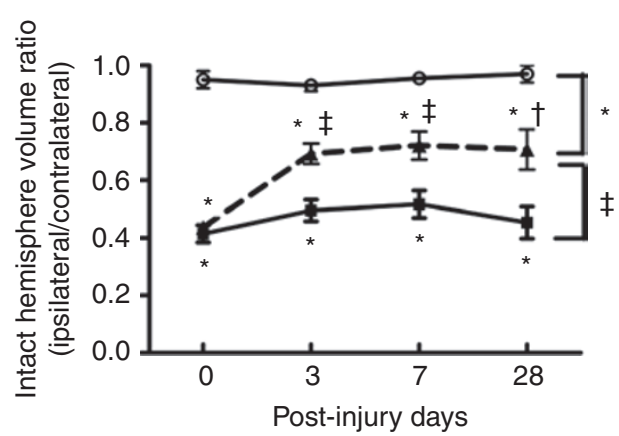

Figure 2. Evolution of brain injury. Representative serial brain MRIs of the NC (left column), MC (middle column), and MM (right column) groups from the medial septal area on (a) day 0 , (b) day 3, (c) day 7, and (d) day 28 after MCAO. The time course of brain injury evolution was evaluated using diffusionweight (DW) and T2-weighted MRI. In vivo T2* MRI was performed to delineate the super-paramagnetic iron oxide (SPIO)-tagged MSCs, which appear as low-signal-intensity areas in T2* of the MM group. (e) The intact brain volume of the ipsilateral hemisphere was measured by MRI at postinjury days $0,3,7$, and 28. Circles represent the NC, squares represent the MC, and triangles represent the MM group. The data are represented as the mean \pm SEM; $n=5,15$, 12 at day $0 ; n=5,14,12$ at day $3 ; n=5,7,11$ at day 7 ; and $n=5,6,11$ at day 28 in the NC, MC, and MM groups, respectively. The general linear model with repetitive measures revealed that each group was different from the others; ${ }^{*} P<0.01$ vs. NC group, ${ }^{\dagger} P<0.05$ vs. MC group, and ${ }^{\ddagger} P<0.01$ vs. MC group. MC, MCAO + PBS-treated group; MCAO, middle cerebral artery occlusion; MM, MCAO + UCB-derived MSC-treated group; MRI, magnetic resonance imaging; MSC, mesenchymal stem cell; NC, sham + PBS-treated group; PBS, phosphate-buffered saline; UCB, umbilical cord blood.

The deposition of PKH26 red fluorescence-positive donor cells was observed only in MM, not in NC or MC. In MM, these cells were especially abundant in the area of the penumbra in close proximity to the peri-infarct area (Figure 4c).

\section{TUNEL Staining and Immunohistochemistry}

As compared with NC, MC had more abundant numbers of TUNEL-positive cells in the peri-infarct area of the rats at $28 \mathrm{~d}$ post-MCAO (P38) (Figure 4d). This MCAO-induced increase in the number of TUNEL-positive cells was significantly attenuated after MSC transplantation (MM, $P<0.05$ vs. MC) (Figure 4g).

The number of GFAP-positive cells was determined by measuring GFAP-immunofluorescence intensity of the peri-infarct area. The intensity was higher in MC and MM as compared with NC (Figure 4e). This increase in the number of GFAPpositive cells was significantly attenuated after MSC transplantation (MM, $P<0.01$ vs. MC) (Figure $4 \mathrm{~h}$ ).

The ED-1-positive cells evaluated in the peri-infarct area were increased in MC as compared with NC (Figure 4f). The number of ED-1-positive cells decreased significantly after MSC transplantation (MM, $P<0.01$ vs. MC) (Figure $4 \mathbf{i}$ ).

\section{Co-labeling of Localized Donor Cells}

A few instances of PKH26 and GFAP or PKH26 and NeuN colocalization were observed, which are suggestive of glial and neuronal differentiation, respectively (Figure 5).

\section{DISCUSSION}

In this study, human UCB-derived MSC transplantation significantly attenuated the increased mortality and growth retardation associated with severe brain injury that involved more than $50 \%$ of the ipsilateral hemisphere volume in the newborn rats. Transplantation also reduced the severity in the changes in brain infarct volume and histologic abnormalities, such as increased astrocytic gliosis, reactive microglia, increased numbers of TUNEL-positive cells, and impaired sensorimotor function. These findings suggest that human UCB-derived MSCs may be a novel therapeutic candidate for severe neonatal brain injury due to neonatal stroke.

Although the response of the immature brain to ischemia differs significantly from that of the adult brain $(15,16)$, there is abundant evidence that inflammation plays an important role in cerebral ischemia in both newborns and adults $(15,17)$. The response to ischemic injury is associated with an acute and prolonged inflammatory process characterized by the activation of resident glial cells and the production of inflammatory cytokines (17). In this study, markedly increased TUNEL-, ED-1-, and GFAP-positive cells were observed in the penumbrae of MCAO rats. These findings suggest that inflammatory responses also play a key role in the brain injury associated with neonatal stroke.

The precise neuroprotective mechanism underlying the beneficial effects of stem cell transplantation has not yet been elucidated. In the adult stroke model, the beneficial effects 

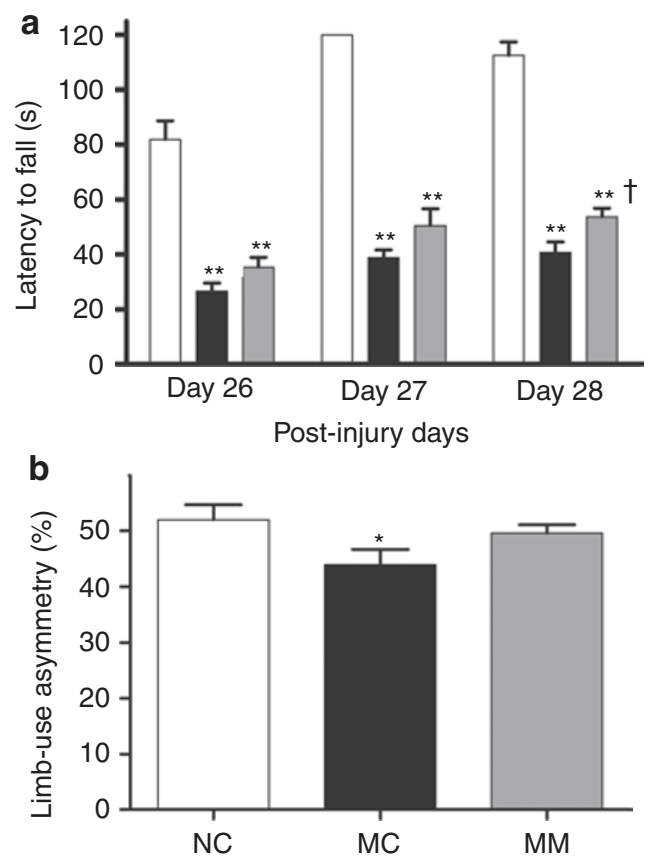

Figure 3. Behavioral tests. (a) Functional outcomes for the rotarod and (b) cylinder tests. White bars represent the NC, black bars represent the MC, and gray bars represent the MM group. The data are represented as the mean \pm SEM; $n=5,5$, and 6 in the NC, MC, and MM groups, respectively; ${ }^{*} P<0.05$ vs. NC group, ${ }^{* *} P<0.01$ vs. NC group, and ${ }^{+} P<0.05$ vs. MC group. $\mathrm{MC}, \mathrm{MCAO}+\mathrm{PBS}$-treated group; MCAO, middle cerebral artery occlusion; MM, MCAO + UCB-derived MSC-treated group; MSC, mesenchymal stem cell; NC, sham + PBS-treated group; PBS, phosphate-buffered saline; UCB, umbilical cord blood.

of stem cells, such as reduction in infarct volumes and neurological deficit, were associated with enhanced endogenous neuronal differentiation, reduced cell death, and inflammatory cytokine attenuation in the injured area (18-22). Trophic factors such as brain-derived neurotrophic factor, vascular endothelial growth factor, and hepatocyte growth factor are thought to mediate the primary neuroprotective mechanism rather than neuronal differentiation $(21,22)$. In addition to a reduction in the infarct volume and improved results on functional tests, MCAO-induced increases in TUNEL-, GFAP-, and ED-1-positive cells in the penumbral area were significantly attenuated by MSC transplantation. However, only a few PKH26-positive MSCs were colabeled with GFAP or NeuN immunostaining, which suggests astroglial or neuronal differentiation of the transplanted MSCs, respectively, in this study. Overall, these findings suggest that the neuroprotective effects of MSCs are primarily mediated or associated with their antiinflammatory effects rather than their regenerative capacities (20-22).

Anti-inflammatory effects mediated by increased expression of trophic factors may be the primary mechanism underlying the effects of engrafted MSCs on injured brain. Previously, we demonstrated an antiinflammatory effect of human UCBderived MSCs on hyperoxic lung injury in newborn rats $(10,11)$. In vivo and in vitro neonatal hypoxia-ischemia studies have also suggested an antiinflammatory influence of MSCs on brain injury as evidenced by reduced microglial activation and by upregulated cytokines such as interleukin-10 (23). Torrente et al.(24) suggested that the beneficial outcomes observed after MSC transplantation into injured brain tissues might include trophic factor delivery and immunomodulation. Thus, MSCs may engage in the formation of a favorable milieu for neurons that mediates antiinflammatory effects by secreting growth factors and cytokines such as vascular endothelial growth factor, hepatocyte growth factor, brain-derived neurotrophic factor, and glial cell line-derived neurotrophic factor $(21,22)$. In the developing brain, the propensity of neurons to undergo cell death is also dependent on the resident inflammatory cell response (15); hence, brain cell death may be modulated by an antiinflammatory milieu induced by MSCs as well as by their regulation of apoptosis (such as caspase-3-dependent cell death) and their stimulation of endogenous cell proliferation and differentiation $(25,26)$. Further studies will be necessary to identify the trophic factors mediating the antiinflammatory responses of an injured brain.

Determining the therapeutic time window is essential for the translation of experimental results into clinical trials. In this study, MSCs were transplanted $6 \mathrm{~h}$ after MCAO because there was a concern that a delay in applying potentially brain-saving treatments beyond the therapeutic window might abolish or drastically reduce its therapeutic effectiveness (27). However, it would be practically impossible to diagnose neonatal stroke and start any therapy within $6 \mathrm{~h}$. Because we have shown the therapeutic efficacy of MSC transplantation against severe MCAO-induced neonatal brain injury in this study, determining the optimal timing of MSC transplantation is the next step of our research.

Determining the optimal route of MSC transplantation is another issue to be addressed for clinical translation. In this study, MSCs were administered intraventricularly because local intraventricular stem cell administration has been shown to be therapeutically more effective than systemic intravenous delivery $(28,29)$. No additional procedures are necessary for intraventricular delivery because the anterior fontanel is open in the newborn infant. Moreover, as cerebrospinal fluid circulates, intraventricular delivery may be replaced by intraspinal transplantation. Further studies will be necessary to confirm the therapeutic efficacy of more practical and less invasive routes of stem cell transplantation such as intraspinal and intranasal (30) delivery.

Apart from improvements in infarct volume and histology, the functional outcome is important for the clinical application of MSCs in treating patients with severe perinatal brain injury. In this study, even with the initial severe brain injury, sensorimotor function was improved in the MSC transplantation group. This result suggests the potential for clinical application. Uncertainty exists about when to examine functional tests in the newborn brain injury with respect to brain maturation stage. We evaluated functional tests at $28 \mathrm{~d}$ postinjury (P38), which is a period similar to the human adolescent period in terms of the stage of brain maturation. We expected this time span to be sufficient for functional recovery $(31,32)$. 

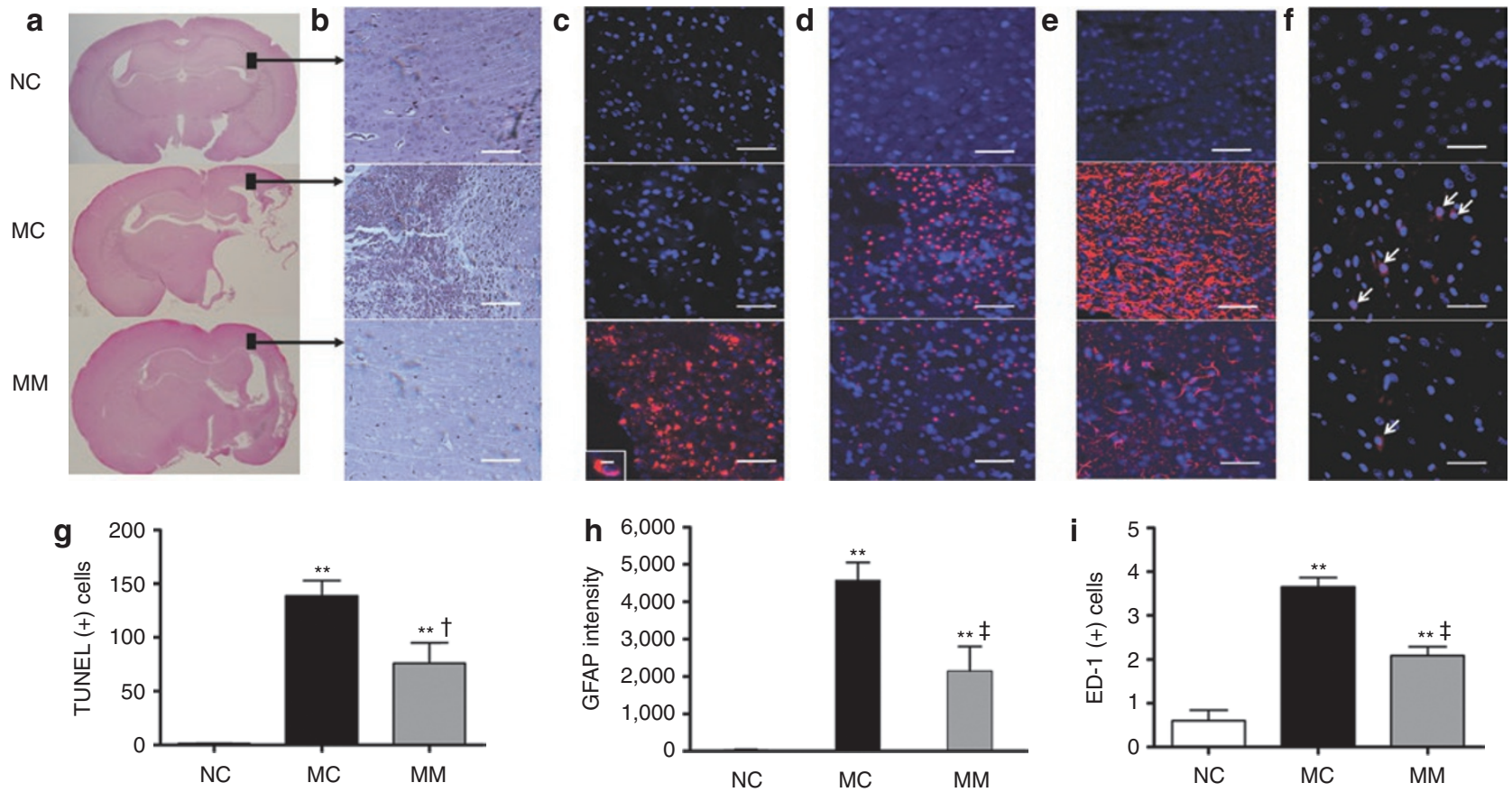

Figure 4. Immunostaining in the ischemic penumbra area of brain. Immunostaining in the penumbra area (black arrows) of each group (a-f).

Photomicrographs of hematoxylin and eosin staining (a) and representative photographs of PKH26-tagged MSCs in the MM group on day 28 after MCAO (b). PKH26-tagging (red) was merged with DAPI staining (blue) (c). TUNEL-positive (red) cells with DAPI staining (blue) (d), glial fibrillary acidic protein (GFAP)-positive (red) cells with DAPI staining (blue) (e), and reactive microglial marker (ED-1)-positive (red) cells (arrows) with DAPI staining (blue) (f) in each group (scale bar $=50 \mu \mathrm{m}$ and $5 \mu \mathrm{m}$ in the magnified photograph). Histograms of TUNEL-positive cells in each group (g). Histogram of GFAP immune-staining intensity in each group (h). Histogram of ED-1-positive cells in each group (i). The data are represented as the mean \pm SEM; $n=4,7$, and 9 in NC, MC, and MM groups, respectively on day 28 after MCAO. ${ }^{* *} P<0.01$ vs. NC group, ${ }^{\ddagger} P<0.01$ vs. MC group, and ${ }^{\dagger} P<0.05$ vs. MC group. DAPI, $4^{\prime}, 6^{\prime}$-diamidino-2-phenylindole dihydrochloride hydrate; MC, MCAO + PBS-treated group; MCAO, middle cerebral artery occlusion; MM, MCAO + UCB-derived MSC-treated group; MSC, mesenchymal stem cell; NC, sham + PBS-treated group; PBS, phosphate-buffered saline; TUNEL, terminal deoxynucleotidyl transferase nick-end labeling; UCB, umbilical cord blood.

a
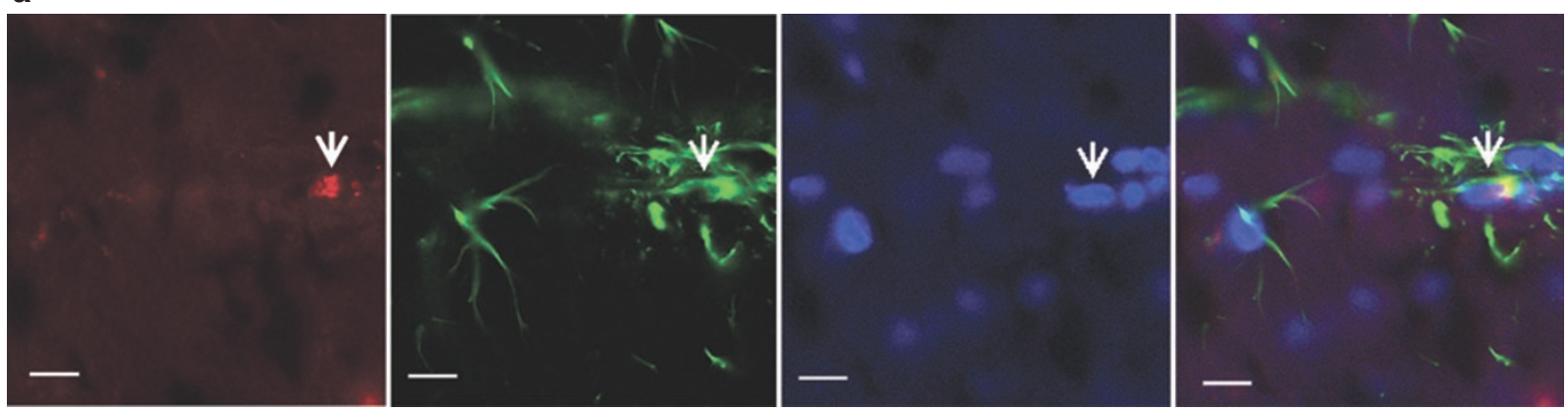

b
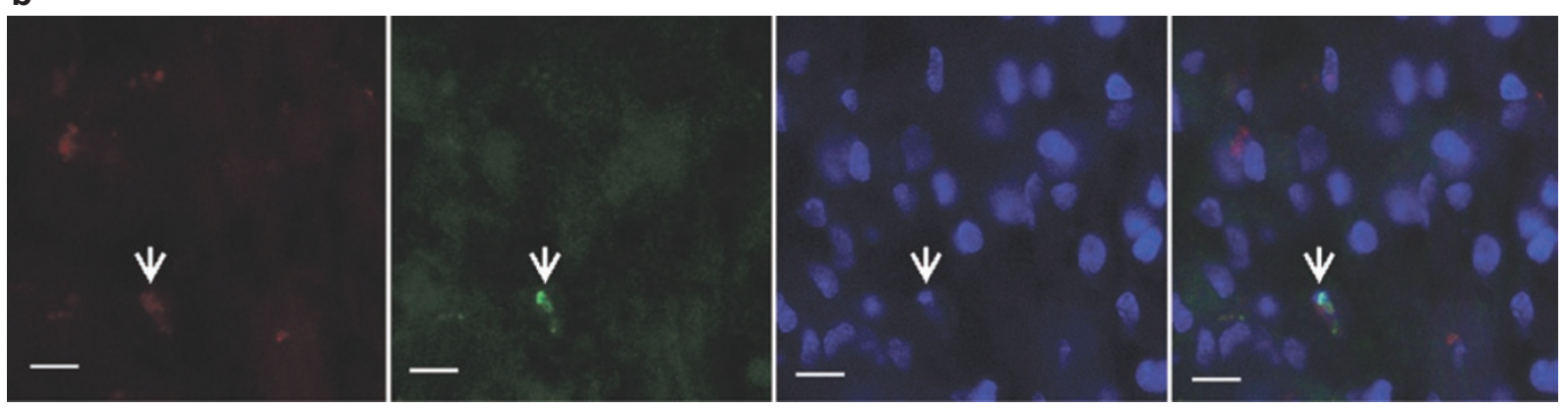

Figure 5. Differentiation of donor cells. (a) GFAP and (b) neuronal-specific nuclear protein (NeuN) stains in the penumbra area with the colocalization of PKH26-tagged MSCs (arrows). Red, PKH26 stain; green, GFAP, or NeuN stain; blue, DAPI stain (scale bar = $20 \mu \mathrm{m}$ ). DAPI, 4',6'-diamidino-2-phenylindole dihydrochloride hydrate; GFAP, glial fibrillary acidic protein; MSC, mesenchymal stem cell. 
To confirm whether the protective effects of MSC transplantation will persist to adulthood, further studies with long-term follow-up will be necessary.

In conclusion, this study suggests that UCB-derived MSCs are a feasible treatment for severe perinatal brain injury in the newborn. The benefit may be due to the antiinflammatory effects mediated by the trophic factors produced by MSCs.

\section{METHODS}

This study was approved by the Institutional Review Board of Samsung Medical Center and by Medipost, Seoul, Korea. All of the experimental protocols were approved by the Institutional Animal Care and Use Committee of Samsung Biomedical Research Institute. The study followed the institutional and National Institutes of Health guidelines for laboratory animal care.

\section{Cell Preparation}

MSCs were isolated and cultivated from human UCB as previously reported $(10,11)$. The UCB was collected from umbilical veins after neonatal delivery with informed consent from the pregnant mothers. The cells were shown to express CD105 and CD73 (99.6 and 96.3\%, respectively) but not CD34, CD45, or CD14 (0.1, 0.2, and $0.1 \%$, respectively). The cells were positive for HLA-AB (96.8\%) but not HLA-DR $(0.1 \%)$, and they expressed pluripotency markers such as Oct- 4 and SSEA-4 (30.5 and 67.7\%, respectively). The human UCBderived MSCs differentiated into various cell types such as respiratory epithelium, osteoblasts, chondrocytes, and adipocytes with specific in vitro induction stimuli. We confirmed the differentiation potential and karyotypic stability of the human UCB-derived MSCs up to the 11 th passage $(10,11)$. In this study, fifth passage human UCB-derived MSCs from a single donor were transplanted.

\section{Permanent Right MCAO Animal Model}

To exclude any gender-related differences in the severity of the brain injury, only male Sprague-Dawley rats at P10 (Orient, Seoul, Korea) were used for the experiments (33). Each rat pup was anesthetized with isoflurane delivered using a nose cone (5\% for induction and a 1.5- to $2 \%$-mixture of air $/ \mathrm{O}_{2}$ (70:30) during the procedure). The rectal temperature was maintained at $37 \pm 1^{\circ} \mathrm{C}$ using an overhead radiant heater and a warming matrix during the procedure. The right common carotid artery was exposed with a midline incision from the anterior neck. MCAO was performed using a monofilament (6-0; silk) precoated with silicone rubber at the tip, which was inserted from the common carotid artery at the bifurcation point of the external carotid artery and the internal carotid artery. The filament was introduced into the internal carotid artery past $1.3 \mathrm{~cm}$ from the external carotid artery-internal carotid artery bifurcation to occlude the right MCA. The filament length of $1.3 \mathrm{~cm}$ from the external carotid artery-internal carotid artery bifurcation was determined after the confirmation of the filament tip position in preliminary studies with P10 rat pups. The pups in the control groups (NC, $n=5$ ) received a sham operation without MCAO. In the sham-operated rat pups, the common carotid artery was exposed in the same way under anesthesia. The filament was left in situ for permanent $\mathrm{MCAO}$, and the skin was closed with sutures. After confirming severe brain injury involving $>50 \%$ ipsilateral to contralateral hemispheric volume ratio as calculated from the initial DWI taken within $6 \mathrm{~h}$ after permanent MCAO, the animals were randomly divided into vehicle (MC, $n=15$ ) and MSC transplantation groups (MM, $n=12$ ).

The animals were then returned to their dams, and fed ad libitum. However, the MCAO rat pups received supplemental feeding four times per d with $1.5-2.0 \mathrm{ml}$ of commercially available formula with a 22 -guage feeding tube until $2 \mathrm{wk}$ after the injury by a blinded animal technician because of poor suckling due to the severe brain injuries.

All animals were weighed daily, and killed at P38, the 28th day after injury.

\section{Transplantation of Donor Cells With Stereotaxis}

Human UCB-derived MSCs from a single donor at the fifth passage were double-labeled using a PKH26GL Red Fluorescent Cell
Membrane Labeling Kit (Sigma-Aldrich, St. Louis, MO) and superparamagnetic iron oxide nanoparticles. The human UCB-derived MSCs were cultured with PKH26GL and ferumoxides (Feridex, I.V.; Taejoon, Seoul, Korea) in culture medium for endocytosis as previously reported (34).

The optimal doses for intraventricular stem cell transplantation are in the range of $3 \times 10^{4}-2.5 \times 10^{5}(23)$. In this study, $1 \times 10^{5}$ cells in 10 $\mu \mathrm{l}$ of PBS ( $\mathrm{pH}$ 7.4) were administered intraventricularly because the maximal concentration of MSCs without adhesion is $1 \times 10^{7} / \mathrm{ml}$. A dose of $10 \mu \mathrm{l}$ is not considered to induce any volume-related effects in the ventricle.

For donor cell transplantation, $1 \times 10^{5}$ cells (MSCs) were administered into the ipsilateral right lateral ventricle using a sterotactic method at $6 \mathrm{~h}$ after permanent MCAO (27). For NC and MC, the same volume of PBS was administered using the same method.

Each rat pup was anesthetized with sodium pentobarbital $(100 \mathrm{mg} / \mathrm{kg})$ before stereotactic intraventricular transplantation. An incision was made through the scalp skin to expose the bregma and the cells or PBS were stereotactically injected at anterior-posterior, $-1.3 \mathrm{~mm}$; medial-lateral, $-0.5 \mathrm{~mm}$; dorsal-ventral, $-3.0 \mathrm{~mm}$. On the basis of preliminary studies of P10 rat pups confirmed by brain MRI, this location was determined to be the middle of the right lateral ventricle. Intraventricular cell transplantation with $10 \mu \mathrm{l}$ volume required $5 \mathrm{~min}$ to prevent backflow; the needle was slowly removed $2 \mathrm{~min}$ after the injection had been completed. After the injection of the MSCs or PBS, the scalp skin was closed with sutures.

\section{In Vivo MRI Assessment}

The MRI was performed while the rats were kept in an anesthetized state by the administration of $1.5-2 \%$ isoflurane in oxygen-enriched air with a facemask.

All MRI examinations were performed using a 7.0-tesla MRI System (Bruker-Biospin, Fällanden, Switzerland) prepared with a $20-\mathrm{cm}$ gradient set capable of providing a rising time of $400 \mathrm{mTm}^{-1}$. The MR images were acquired with $1.0-\mathrm{mm}$ slice thickness, and a total of 12 slices were acquired with the following parameters: for DWI: repetition time $=3,000 \mathrm{~ms}$, time to echo $=28.5 \mathrm{~ms}$, b-factor $=1,000 \mathrm{~s} /$ $\mathrm{mm}^{2}$, field of view $=25.6 \mathrm{~mm} \times 25.6 \mathrm{~mm}$, matrix size $=256 \times 256$, number of excitations $=1$; for T2-weighted imaging: repetition time $=3,000 \mathrm{~ms}$, time to echo $=60 \mathrm{~ms}$, field of view $=25.6 \mathrm{~mm} \times 25.6 \mathrm{~mm}$, matrix size $=256 \times 256$, number of excitations $=12$; for T $2 *$-weighted imaging: repetition time $=208 \mathrm{~ms}$, time to echo $=10 \mathrm{~ms}$, flip angle $=15^{\circ}$, field of view $=25.6 \mathrm{~mm} \times 25.6 \mathrm{~mm}$, matrix size $=256 \times 256$, number of excitations $=12$.

MRI was performed at $1 \mathrm{~h}$ and $6 \mathrm{~h}$ (immediately after MSC or PBS transplantation) and on the 3rd (P13), 7th (P17), and 28th (P38) day post-MCAO. One MRI session was on average $30 \mathrm{~min}$ per pup and all sessions included DWI, T2-weighted imaging, and T2*-weighted imaging for both the human UCB-derived MSC and PBS transplantation groups. After the MRI exams, the rat pups were allowed to recover and were returned to their dams.

\section{Measurement of the Extent of Brain Injury by MRI}

All MR images were analyzed using ParaVision 2.0.2 software (Bruker BioSpin, Karlsruhe, Germany). The lesion was well identified by the hyperintense areas in DWI at $1 \mathrm{~h}, 6 \mathrm{~h}$, and the $3 \mathrm{rd}$ day post-MCAO (P10 and P13) and by the hyperintense areas in T2-weighted imaging on the 7th and 28th days post-MCAO (P17 and P38). The ipsilateral to contralateral hemispheric intact brain volume ratio was calculated as a parameter of brain injury. For each image, two blinded examiners manually calculated the intact brain ratio by outlining the images. The values calculated by the two examiners were averaged and used as final outcomes.

\section{Rotarod Test}

To evaluate sensorimotor function, rotarod tests were performed by analyzing the latency to fall on the 26th, 27th, and 28th day postMCAO (P36, P37, and P38, respectively). The rotation speed of the treadmill was accelerated from 4-40 rpm over $100 \mathrm{~s}$ for a maximum of $2 \mathrm{~min}$. All animals were tested three times for the three consecutive days. There was a 15-min interval between trials. Because rats have the ability to learn the rotarod tests, the values were analyzed by date 
and the average latency to fall from the three trials was used as the result.

\section{Cylinder Test}

Cylinder tests were performed by analyzing forelimb movement within a transparent cylinder $(25 \mathrm{~cm}$ diameter and $40 \mathrm{~cm}$ height $)$ with two mirrors positioned behind the cylinder. The first limb to contact the wall was scored as an independent wall placement for that limb. If an animal placed both paws on the wall, both limbs were scored. Each animal was video-recorded for 5 min during each session. Limbuse asymmetry scores were calculated using the following formula: number of (left forelimb contacts $+1 / 2$ both contacts) $/$ (right forelimb contacts + left forelimb contacts + both contacts). All measurements were recorded three times on the 28th day post-MCAO (P38) for each animal. "Inactive" trials ( $<10$ wall movements per session) were excluded from the calculation.

\section{Tissue Preparation}

Brain tissue preparation procedures were performed in the surviving animals until $28 \mathrm{~d}$ post-MCAO (P38). The animals were anesthetized with sodium pentobarbital $(100 \mathrm{mg} / \mathrm{kg})$, and their brains were isolated after thoracotomy and transcardiac perfusion with ice-cold $4 \%$ paraformaldehyde in $0.1 \mathrm{~mol} / \mathrm{l} \mathrm{PBS}$. The brains were cautiously removed from the animals and fixed overnight with $4 \%$ formaldehyde solution at room temperature $(n=4,7$, and 9 in the NC, MC, and MM groups, respectively) or frozen at $-70^{\circ} \mathrm{C}$ ( $n=1$ for each group). The brains were embedded in paraffin, and coronal serial sections $(4 \mu \mathrm{m}$ thick) were taken from the paraffin blocks for morphometric analyses, or they were sectioned coronally $(10 \mu \mathrm{m}$ thick) on a cryostat (30510 S, Leica CM, Nussloch, Germany) at the level of the medial septum area $(+0.95 \mathrm{~mm}$ to -0.11 /bregma) and the hippocampal area $(-2.85$ to $-3.70 \mathrm{~mm}$ ). Sections (thickness of $4 \mu \mathrm{m}$ each) were stained with hematoxylin and eosin to assess the extent of neuronal damage. Three sections including penumbra were chosen for each rat, and three random microscopic fields of penumbra were evaluated by a blinded observer.

\section{Immunohistochemistry}

Cryosections ( $10 \mu \mathrm{m}$ thick) were mounted with Vectashield mounting solution containing $4^{\prime}, 6^{\prime}$-diamidino-2-phenylindole dihydrochloride hydrate (H-1200; Vector, Burlingame, CA). The MSCs were immunostained using PKH26 red fluorescence; the $40 \times$ (and 120×) objective images were also stained with $4^{\prime}, 6^{\prime}$-diamidino-2-phenylindole dihydrochloride hydrate to detect nuclear signals. The sections were analyzed by confocal microscopy (Biorad Radiance 2100; Bio-Rad, Hercules, CA).

Immunofluorescence histochemistry was performed for GFAP (rabbit polyclonal; Dako, Glostrup, Denmark) as the astrocytic glial marker, NeuN (mouse monoclonal; Chemicon, Temecula, CA) as the neuron-specific marker, and ED-1 (mouse monoclonal; Millipore, Concord Road, MA) as the marker for reactive microglia.

The slices were incubated with the primary GFAP antibody (overnight, room temperature, 1:1,000 in PBS with $1 \%$ bovine serum albumin). After three rinses (same buffer), the sections were subsequently incubated with Alexa Fluor 568 (red) or 488 (green) conjugated anti-rabbit immunoglobulin (90 min, diluted 1:200; Molecular Probes, Eugene Oregon). After three rinses, the sections were mounted with Vectashield mounting solution containing $4^{\prime}, 6^{\prime}$ diamidino-2-phenylindole dihydrochloride hydrate and visualized by $40 \times$ confocal microscopy (Biorad Radiance 2100; Bio-Rad). The number of GFAP-positive cells was determined by a blinded observer in three nonoverlapping random fields of penumbra cortex per animal. Immunostaining with primary NeuN antibody and ED-1 (both 1:100 dilutions) was performed with the same procedure as described above.

\section{TUNEL Staining}

The immunofluorescent terminal TUNEL technique (kit S7110 ApopTag; Chemicon) was used to determine apoptosis levels in the sections according to the manufacturer's protocol. The slides were mounted with Vectashield mounting solution with $4^{\prime}, 6^{\prime}$-diamidino2 -phenylindole dihydrochloride hydrate (H-1200; Vector) and visualized by $40 \times$ confocal microscopy (Biorad Radiance 2100; Bio-Rad).
The number of TUNEL-positive cells in three nonoverlapping random fields for each animal was determined by a blinded observer.

\section{Statistical Analyses}

The data were expressed as the mean \pm SEM of multiple independent experiments. Survival rates were compared by Kaplan-Meier analysis followed by a log-rank test. For time-course variables, a general linear model with repetitive measures was analyzed with a Bonferroni correction. For continuous variables with a normal distribution, the groups were compared using Student's $t$-test with a Bonferroni correction. Continuous variables that did not show a normal distribution were analyzed by Wilcoxon rank test with a Bonferroni correction. All of the data were analyzed using SPSS version 12.0 (SPSS, Chicago, IL). Values of $P<0.05$ were considered statistically significant.

\section{ACKNOWLEDGMENTS}

We are grateful to Dong Kyeom Yoo for performing copious MRI scanning work.

\section{STATEMENT OF FINANCIAL SUPPORT}

This work was supported by a Korea Research Foundation grant from the Korean Government (MOEHRD, Basic Research Promotion Fund; grant S-20110272-000), a grant from the Korean Healthcare Technology R\&D Project, Ministry for Health, Welfare \& Family Affairs, Republic of Korea (grant A110445), and by a grant from the Sungkyunkwan University Foundation for Corporate Collaboration (grant S-2010-032-000).

\section{REFERENCES}

1. Kirton A, deVeber G. Advances in perinatal ischemic stroke. Pediatr Neurol 2009;40:205-14.

2. Mineyko A, Kirton A. The black box of perinatal ischemic stroke pathogenesis. J Child Neurol 2011;26:1154-62.

3. Derugin N, Ferriero DM, Vexler ZS. Neonatal reversible focal cerebral ischemia: a new model. Neurosci Res 1998;32:349-53.

4. Wen TC, Rogido M, Gressens P, Sola A. A reproducible experimental model of focal cerebral ischemia in the neonatal rat. Brain Res Brain Res Protoc 2004;13:76-83.

5. Pabello NG, Tracy SJ, Keller RW Jr. Protective effects of brief intra- and delayed postischemic hypothermia in a transient focal ischemia model in the neonatal rat. Brain Res 2004;995:29-38.

6. Harbert MJ, Tam EW, Glass HC, et al. Hypothermia is correlated with seizure absence in perinatal stroke. J Child Neurol 2011;26:1126-30.

7. Wyatt JS, Gluckman PD, Liu PY, et al.; CoolCap Study Group. Determinants of outcomes after head cooling for neonatal encephalopathy. Pediatrics 2007;119:912-21.

8. Lin Y, Ko T, Shih Y, et al. Human umbilical mesenchymal stem cells promote recovery after ischemic stroke. Stroke 2011;42:2045-53.

9. Banerjee S, Williamson D, Habib N, Gordon M, Chataway J. Human stem cell therapy in ischaemic stroke: a review. Age Ageing 2011;40:7-13.

10. Chang YS, Choi SJ, Sung DK, et al. Intratracheal transplantation of human umbilical cord blood derived mesenchymal stem cells dose-dependently attenuates hyperoxia-induced lung injury in neonatal rats. Cell Transplant, e-pub ahead of print 7 March 2011.

11. Chang YS, Oh W, Choi SJ, et al. Human umbilical cord blood-derived mesenchymal stem cells attenuate hyperoxia-induced lung injury in neonatal rats. Cell Transplant 2009;18:869-86.

12. Kim ES, Chang YS, Choi SJ, et al. Intratracheal transplantation of human umbilical cord blood-derived mesenchymal stem cells attenuates Escherichia coli-induced acute lung injury in mice. Respir Res 2011;12:108.

13. ClinicalTrials.gov. Oh W (MD): Safety and efficacy evaluation of Pneumostem treatment in premature infants in bronchopulmonary dysplasia. (http://www.clinicaltrials.gov/ct2/show/NCT01297205?term=NCT01297 205\&rank=1)

14. ClinicalTrials.gov. Cotten CM (MD): Cord blood for neonatal hypoxicischemia encephalopathy. (http://www.clinicaltrials.gov/ct2/show/NCT00 593242? term=NCT00593242\&rank=1).

15. Vexler ZS, Yenari MA. Does inflammation after stroke affect the developing brain differently than adult brain? Dev Neurosci 2009;31:378-93.

16. Yager JY, Thornhill JA. The effect of age on susceptibility to hypoxic-ischemic brain damage. Neurosci Biobehav Rev 1997;21:167-74. 
17. Benjelloun N, Renolleau S, Represa A, Ben-Ari Y, Charriaut-Marlangue C. Inflammatory responses in the cerebral cortex after ischemia in the P7 neonatal Rat. Stroke 1999;30:1916-23; discussion 1923-4.

18. Dharmasaroja P. Bone marrow-derived mesenchymal stem cells for the treatment of ischemic stroke. J Clin Neurosci 2009;16:12-20.

19. Yoo SW, Kim SS, Lee SY, et al. Mesenchymal stem cells promote proliferation of endogenous neural stem cells and survival of newborn cells in a rat stroke model. Exp Mol Med 2008;40:387-97.

20. Bonfield TL, Nolan Koloze MT, Lennon DP, Caplan AI. Defining human mesenchymal stem cell efficacy in vivo. J Inflamm (Lond) 2010;7:51.

21. Doorn J, Moll G, Le Blanc K, van Blitterswijk C, de Boer J. Therapeutic applications of mesenchymal stromal cells: paracrine effects and potential improvements. Tissue Eng Part B Rev 2012;18:101-15.

22. Choi YJ, Li WY, Moon GJ, et al. Enhancing trophic support of mesenchymal stem cells by ex vivo treatment with trophic factors. J Neurol Sci 2010;298:28-34.

23. Pimentel-Coelho PM, Mendez-Otero R. Cell therapy for neonatal hypoxic-ischemic encephalopathy. Stem Cells Dev 2010;19:299-310.

24. Torrente Y, Polli E. Mesenchymal stem cell transplantation for neurodegenerative diseases. Cell Transplant 2008;17:1103-13.

25. Chen J, Li Y, Katakowski M, et al. Intravenous bone marrow stromal cell therapy reduces apoptosis and promotes endogenous cell proliferation after stroke in female rat. J Neurosci Res 2003;73:778-86.

26. van Velthoven CT, Kavelaars A, van Bel F, Heijnen CJ. Regeneration of the ischemic brain by engineered stem cells: fuelling endogenous repair processes. Brain Res Rev 2009;61:1-13.
27. Park WS, Sung DK, Kang S, et al. Therapeutic window for cycloheximide treatment after hypoxic-ischemic brain injury in neonatal rats. J Korean Med Sci 2006;21:490-4.

28. Li L, Jiang Q, Ding G, et al. Effects of administration route on migration and distribution of neural progenitor cells transplanted into rats with focal cerebral ischemia, an MRI study. J Cereb Blood Flow Metab 2010;30:653-62.

29. Mueller D, Shamblott MJ, Fox HE, Gearhart JD, Martin LJ. Transplanted human embryonic germ cell-derived neural stem cells replace neurons and oligodendrocytes in the forebrain of neonatal mice with excitotoxic brain damage. J Neurosci Res 2005;82:592-608.

30. van Velthoven CT, Kavelaars A, van Bel F, Heijnen CJ. Nasal administration of stem cells: a promising novel route to treat neonatal ischemic brain damage. Pediatr Res 2010;68:419-22.

31. Quairiaux C, Sizonenko SV, Mégevand P, Michel CM, Kiss JZ. Functional deficit and recovery of developing sensorimotor networks following neonatal hypoxic-ischemic injury in the rat. Cereb Cortex 2010;20:2080-91

32. Lee JA, Kim BI, Jo $\mathrm{CH}$, et al. Mesenchymal stem-cell transplantation for hypoxic-ischemic brain injury in neonatal rat model. Pediatr Res 2010;67:42-6.

33. Lang JT, McCullough LD. Pathways to ischemic neuronal cell death: are sex differences relevant? J Transl Med 2008;6:33.

34. Lee JK, Lee MK, Jin HJ, et al. Efficient intracytoplasmic labeling of human umbilical cord blood mesenchymal stromal cells with ferumoxides. Cell Transplant 2007;16:849-57. 